\title{
Battle of COVID-19: Where We Can Take The Help Of Machine Learning To Win?
}

\author{
Wathiq Mansoor ${ }^{1}$ Yashbir Singh ${ }^{2}$
}

1 University of Dubai, UAE

2 Mayo Clinic, Rochester, Mn, USA

\begin{abstract}
COVID-19 is caused by Coronavirus which has been previously linked with 2012 SARS disease. This is an RNA virus. Highly contagious is nature; spreads by air droplet and contact. It needs both air and contact precautions. Interventions to prevent its spread have been tried which includes social distancing, preventing skin contact with the patient and handwashing with soap. Use of sanitizers with $>60 \%$ alcohol content has been useful. In scientific methods including pre-detection of signs and symptoms help to prevent the spread by quarantining the sick from the healthy for a period of at least 2 weeks. We can use the technology known to us; here, in this short communication, we have focused to show the basic picture of updated technology which is Artificial Intelligence.
\end{abstract}

Keywords: Pandemic era, COVID19, Artificial intelligence, Deep learning, Radiology, Robotics.

\section{INTRODUCTION}

Nowadays, COVID-19, a novel coronavirus pandemic has disrupted our society in many ways. Digital healthcare innovations are required more than ever used before as we come across myriad challenges during these pandemics. Scientists and developers are learning and finding a way to use artificial intelligence applications and natural language processing to comprehend and tackle this disease. In this COVID-19 era, we do not have any vaccine approved for this disease so far, and the efforts involved to limit the spread of this disease is by using very common strategies such as case identification and contact isolation, encouraging social distancing practices in the population as well as a lockdown of the region to contain the cases and possibly exposed people. The virus compelled almost all of us indoors and pressured us to brainstorm ideas to best use science and technology to prevent hospitalizations and mortality. Various members of academic institutions and scientific societies including the leaders of Machine Learning (ML) societies came forward to help. There have been scientific blogs and articles on COVID-19 and use of ML, among them, many of them have offered very comprehensive views so far. Machine learning may help to reduce the impacts of this pandemic in conjunction with all the research and strategies going on.

Here, we are presenting the few possible approaches to aid this pandemic:

\subsection{ML to identify drug compounds and generate a model}

Machine learning can help in advance drug development process providing a deeper understanding of clinical effects, can predict infection rates, and help in the faster screening of drug compounds (Zhang, et al.). There are various other suitable application areas but the problem is limited data sharing in healthcare due to privacy issues and the ability to combine composite structures into Deep Learning models.

- Drug screening using Deep Learning approach for COVID-19 by (Zhang, et al.) has developed a model to rapidly screen thousands of drug compounds database and identify RNA sequences and useful compounds which are stable, which has more bioavailability and which develop less immune reactions. A list of compounds and tripeptides found may help to develop antiviral drugs for the 2019nCov_main_protease of COVID-19 patients, a main therapeutic target.

- AlphaFold is a deep learning library for computational chemistry to predict a protein structure in SARS-CoV-2 using amino acid sequences. It may help scientists to find a close molecular structure of the COVID-19 virus which could potentially pave the way for finding a vaccine for this devastating infection. 


\subsection{ML in Diagnostic Radiology}

The researchers have taken the help of AI accurately diagnose COVID-19 on CT scans and to distinguish it from community acquired pneumonia. Chen et al research have developed a deep learning method for detecting COVID-19 in CT modalities and the result showed high accuracy for a test. This deep learning concept on imaging could help minimize the burden on radiologists and physicians. However, they also warned that the AI tools are based on limited data available at this time for this novel virus [1,2]. Using the radiology, researchers found heart involvement with COVID-19 is very common and has been seen in 23\% of the patient with COVID infection. Cardiac involvement is in terms of myocarditis (inflammation of the heart muscle), heart failure (with reduced heart function) and myocardial infarction (cardiac injury). There could be involvement in terms of arrhythmias in the patient. Correlation of the findings with history of possible exposure and sign with symptoms can help to presume diagnose early.

\subsection{Automation in $3 D$ printing}

As we know, the healthcare systems are completely overloaded and fatigued by COVID-19. The production of medical devices is a complicated process. Manufacturers and developers of medical products have already begun to help the global crisis by using their resources to ease the pressure on supply chains. 3D printing industries also came forward to make 3D printed face shields, masks, goggles respirator valves, and even ventilators. The scope and utility of the 3D printing technology are highly commendable and AI can be incorporated to aid in production. Automation in software can be set up to improve 3D printing workflow to maximize the production of face shields and respiratory valves with the best possible way to utilize resources [3].

\subsection{Internet of Things (IoT) and ML}

A very exciting way to use IoT platforms is to use it to improve the quality of air in the environment. There are especially few possible technologies so far that works behind the IoT technology from improving the air quality. This way the IoT platforms can be used to prevent the spread of the virus that is easily transmitted by air. Inexpensive IoT sensors can be distributed throughout the societies that could monitor the air quality in the local areas. A cloud-based AI platform measures the data collected from sensors and decides the overall air quality [4]. In the other aspect, IoT plays very important role in the virtual meetings. Zoom, Face Time, and Skype are getting very popular because they connect the people to remotely communicate with machines for virtual inspections and remote diagnostic support as well.

\subsection{Service with Intelligent Robot}

The robots, trained with automation using the principals of artificial intelligence can be used to replace human workers during this crisis to help companies in manufacturing and delivering personal protective equipment (PPEs) . It would be very amazing if we could protect our doctors and nurses by programming some work on robots. Moreover, robots could also facilitate a form of telemedicine such as screening the incoming patients for risk by collecting preliminary data for symptoms and exposure and keep the individuals out of areas of infection. This intelligence robotic systems can be speedily deployed and operated by expert healthcare professionals remotely without traveling. Imagine how convenient and safe a future will become in which AI-enabled robot brings food and materials to isolated individual's homes $[5,6]$.

\subsection{Personal Protective Equipment (PPE) manufacturing optimization with $M L$}

Many medical professionals over the world have complained about the shortage in supply of PPEs due to rising demand, panic buying, and misuse of them in the current pandemic situation. PPE is the main apparatus by which health professionals protect themselves and their patients from being infected and infecting others. The common type of PPE are gloves, medical face mask (N95 or FFP2 standard or equivalent), eye protection goggles, gown, aprons, and boots covering film. They are made with porous and non-porous polymeric film. The porous polymeric films are mainly used for the production of medical face mask, gown, and aprons as they are permeable to air but reject the particle size of coronavirus. Currently, the melt spin manufacturing technology is used for the production of polyethylene, or polypropylene fibers are used for the production of the porous film. The technology such as electrospinning which has the advantage of the porosity than melt spin porous film is also used for the production. The non-porous polymeric film used for the manufacturing of other PPE materials such as face shield gloves and boots covering film is made with a different type of molding method [7]. The different polymers and their additives are added into the molding to achieve the proper mechanical properties of the final product $[8,9,10,11,12]$. 3-D printing is also a good method for production of the PPE but it impacts the manufacturing rate.

In the current scenario, the main goal of many PPE manufacturing industry is to increase the production of material without impacting the quality of them. The industry has enough data about how different process parameters impact the outcomes. Due to the complexity in the production, there is a requirement of proper computing platforms such as process-based machine learning which easily analyzes the outcomes and changes the automation accordingly. 


\subsection{Deep reinforcement learning to explore environmental factors and impact on public health}

This is an artificial intelligence approach that explores the environment and receives actions based on rewards which are defined already in the environment. This approach could work more closely in the battle against COVID-19 spread. In this actual harsh time that everyone knows, we need to learn from the environment how to take wise actions against this COVID-19. In this kind of study, we have to understand the ratio of the cost of our decisions, considering a multitude of factors like local and global economic impact, public health, and psychological effects. These types of study address Reinforcement Learning, especially integrating it with Deep Neural Networks that is called deep reinforcement learning [13, 14].

\subsection{ML in downstream analysis of sequencing data}

Next generation sequencing (NGS) has become a most popular technique in the field of Genomics. The quality of NGS varies widely with read depth, reference sequences, mapping accuracy, and used pipeline. For analysis of sequencing data through Machine Learning is the recent popular approach to identify Differential expressed genes (DEGs). Due to features of ML algorithm, it could be utilised for analysis of DEGs in accuracy manner. Basic analysis steps are classified into following: 1) Preprocessing feature of data 2) Sequencing feature 3) Downstream feature for analysis.

\section{LIMITATIONS}

Limited data sharing continues to inhibit the entire progress in medical research problems. We believe that utilizing things like meta-learning, domain adaptation, and reinforcement learning while loosening restrictions on healthcare data, could allow Machine Learning to play a very significant role in containing and responding to both COVID-19 and future pandemics.

\section{CONCLUSION}

We recommend the widespread use of artificial intelligence principles and applications to fight against the Covid-19 as the most potentially impactful ML approaches are still not utilised widely as they should be.

\section{CONFLICTS OF INTEREST: None}

distinguishes covid-19 from community acquired pneumonia on chest ct. Radiology, 200905

[2] Singh, Y., Shakyawar, D., \& Hu, W. (2020). An Automated Method for Detecting the Scar Tissue in the Left Ventricular Endocardial Wall Using Deep Learning Approach. Current Medical Imaging, 16(3), 206-213.

[3] Pearce, J. M. (2020). A review of open source ventilators for COVID-19 and future pandemics. F1000Research, 9(218), 218.

[4] Dutta, J., Chowdhury, C., Roy, S., Middya, A. I., \& Gazi, F. (2017, January). Towards smart city: sensing air quality in city based on opportunistic crowd-sensing. In Proceedings of the 18th international conference on distributed computing and networking (pp. 1-6).

[5] Huang, C. J., Chen, Y. H., Ma, Y., \& Kuo, P. H. (2020). Multiple-Input Deep Convolutional Neural Network Model for COVID-19 Forecasting in China. medRxiv.

[6] Yang, G. Z., Nelson, B. J., Murphy, R. R., Choset, H., Christensen, H., Collins, S. H., ... \& Kragic, D. (2020). Combating COVID-19- The role of robotics in managing public health and infectious diseases.

[7] Ultraporous nanofiber mats and uses thereof; UCN104936671A, JP2017200940A US14648925, EP2928577A1, EP2928577A4, WO2014093345A1, Patents. crosslinked epoxy using low field NMR spin lattice relaxometry; Journal of Polymer Science PartB:PolymerPhysics2018, 56, 639.

[8] Patel, J. P., Zou, G., Hsu, S.L, et al.; Path to achieving molecular dispersion in a dense reactive mixture; Journal of Polymer Science Part B: Polymer Physics 2015, 53, 1519-1526.

[9] Patel, J. P., Hsu, S.L, et al.; An analysis of the role of non-reactive plasticizers in the crosslinking reactions of phenolic resins; Journal of Polymer Science Part B:Polymer Physics 2016,55,206-213.

[10] Singh, Y., Wu, S. Y., Friebe, M., Tavares, J. M. R., \& Hu, W. (2018). Cardiac Electrophysiology Studies Based on Image and Machine Learning.

[11] Singh, Y., Shakyawar, D., \& Hu, W. (2020). Nonischemic endocardial scar geometric remodeling toward topological machine learning. Proceedings of the Institution of Mechanical Engineers, Part H: Journal of Engineering in Medicine, 234(9), 1029. 103

\section{REFERENCES}

[1] Li, L., Qin, L., Xu, Z., Yin, Y., Wang, X., Kong, B., ... \& Cao, K. (2020). Artificial intelligence 